\title{
Latency and Jitter Analysis for IEEE 802.11e Wireless LANs
}

\author{
Sungkwan Youm ${ }^{1}$ and Eui-Jik Kim ${ }^{2}$ \\ ${ }^{1}$ Telecommunication Systems Division, Samsung Electronics Co., Ltd., Dong Suwon P.O. Box 105, 416 Maetan-3dong, \\ Yeongtong-gu, Suwon-si, Gyeonggi-do 443-742, Republic of Korea \\ ${ }^{2}$ Department of Ubiquitous Computing, Hallym University, 39 Hallymdaehak-gil, Chuncheon-si, Gangwon-do 200-702, \\ Republic of Korea
}

Correspondence should be addressed to Eui-Jik Kim; ejkim32@hallym.ac.kr

Received 24 September 2013; Accepted 31 October 2013

Academic Editor: Jongsung Kim

Copyright ( $) 2013$ S. Youm and E.-J. Kim. This is an open access article distributed under the Creative Commons Attribution License, which permits unrestricted use, distribution, and reproduction in any medium, provided the original work is properly cited.

\begin{abstract}
This paper presents a numerical analysis of latency and jitter for IEEE 802.11e wireless local area networks (WLANs) in a saturation condition, by using a Markov model. We use this model to explicate how the enhanced distributed coordination function (EDCF) differentiates classes of service and to characterize the probability distribution of the medium access control (MAC) layer packet latency and jitter, on which the quality of the voice over Internet protocol (VoIP) calls is dependent. From the proposed analytic model, we can estimate the available number of nodes determining the system performance, in order to satisfy user demands on the latency and jitter.
\end{abstract}

\section{Introduction}

The widespread implementation of IEEE 802.11 as the standard for wireless local area networks (WLANs) supports the notion that WLANs may soon be widely adopted for multiservice communication networks. The primary impediment to the use of IEEE 802.11 in multiservice wireless networks is the lack of quality-of-service $(\mathrm{QoS})$ functionality that is required by real-time voice and video applications [1-7]. To resolve this problem, the IEEE 802.11 Working Group created Task Group E to design medium access control (MAC) layer QoS enhancements to the IEEE 802.11 standard [8]. The focus of the IEEE 802.11e standard is the hybrid coordination function (HCF), which provides an efficient mechanism for centrally coordinated medium access, and uses the enhanced distributed coordination function (EDCF) for distributed coordination of medium access. EDCF provides service differentiation between different traffic priorities and is backward compatible with the legacy 802.11 DCF [9].

To analyze and compute the IEEE 802.11 DCF saturated throughput, a Markov chain model for the binary exponential back-off procedure is considered in [10], and in [11, 12] the authors attempted to construct a mathematical model for the throughput performance of 802.11e, even though they did not present the latency and jitter analysis of a packet. In addition, the average delay of IEEE 802.11e is computed in [11], by using Markov chain analysis. A more practical queuing model incorporating practical packet arrival processes is considered under the nonsaturated environment for IEEE 802.11 DCF in [13]. In [14-16], a personal area network, which is operated under IEEE 802.15.4 and on a DCF-similar mechanism in the nonsaturated scenario, is analyzed, using the theory of discrete time Markov chains and M/G/1/K queues. These models that are proposed for analysis of IEEE 802.11e require complexity, which leads the computation power for admission control in IEEE 802.11e. In [17], a model is proposed to address this issue on throughput and delay analysis, except the jitter.

Since the crucial factors in future QoS networks are latency and jitter, the analysis of delay and therefore the latency and jitter that determines this have to be investigated. To our best knowledge, there is no comprehensive study on the characteristic analysis of latency and jitter for IEEE 802.11e WLANs, and delay analysis is limited to the derivation of the mean value; therefore, we need to analyze the latency and jitter by using higher moments, and the probability distribution function of the delay. 
In this paper, we present an analysis of latency and jitter, by using a Markov model for IEEE 802.11e in the saturation condition. This model is used to elucidate how the EDCF differentiates service. We characterize the probability distribution of the MAC layer packet latency and jitter. In this paper, the latency is the time interval between the time instant a packet starts to contend for transmission and the time instant that either successful reception of the packet by the intended receiver is acknowledged, or the packet is dropped. The jitter is the interarrival time between successful packet transmissions of the station and is obtained from the standard deviation of the latency.

The outline of this paper is as follows. The saturation Markov model is shown in Section 2. The analytical model for the packet latency and jitter is developed in Section 3. Comparisons of analytical and simulation results are presented in Section 4. The conclusions are given in Section 5.

\section{EDCF Analytical Model}

A simple and accurate analytical model was presented to compute the throughput of a saturated IEEE 802.11e DCF network under ideal channel conditions [11]. The model relies on two discrete time processes, to model the progress of a given station through back-off. One process $b(t)$ represents the back-off counter of the station, which is decremented at the end of every idle back-off slot. When it reaches zero, the station transmits. Regardless of the transmission result, it starts a new back-off procedure, by drawing a new value for $b(t)$. It only monitors back-off slots, and the decrement of the back-off counter is suspended for the duration of all transmissions and interframe spaces (i.e., SIFS and AIFS). Since the value of $b(t)$ after transmissions depends on the size of the contention window from which it is drawn, and the contention window increases after collision, $b(t)$ depends on the station's transmission history and is therefore non-Markovian. To overcome this problem, another process, $s(t)$, called a stage, is defined, to track the size of the contention window $\left(W[i]_{j}, j=s(t)\right)$. After every successful transmission, $s(t)$ is reset to zero, and for each collision, it is incremented by one, up to a maximum of $m$. We assume that the probability a station in class $i$ will attempt transmission in a timeslot is a constant $\tau[i]$, across all timeslots. In addition, we assume that the probability a packet collision in class $i$ will be experienced by any transmission is a constant $p_{c[i]}$ and is independent of the number of collisions previously experienced. Using these assumptions, a multidimensional Markov process $\{(s(t), b(t))\}$ can be formulated. We describe the Markov chain model of EDCF under saturation condition in Figure 1.

The corresponding state space of the model is

$$
\Omega=\left\{(j, k) \mid 0 \leq j \leq m, 0 \leq k \leq W[i]_{j}-1, j=0, \ldots, m\right\},
$$

and the one-step transition probabilities of the Markov chain are given by

$$
\begin{gathered}
P\{j, k \mid j, k+1\}=1, \quad j \in[0, m], \\
k \in\left[0, W[i]_{j}-1\right], \\
P\{j, k \mid j-1,0\}=\frac{p_{c[i]}}{W[i]_{j}}, \quad j \in[0, m), \\
k \in\left[0, W[i]_{j}-1\right], \\
P\{0, k \mid i, 0\}=\frac{\left(1-P_{c[i]}\right)}{W[i]_{0}}, \quad j \in[0, m), \\
\quad k \in\left[0, W[i]_{i}-1\right], \\
P\{0, k \mid m, 0\}=\frac{1}{W_{[i]_{0}}}, \quad k \in\left[0, W[i]_{0}-1\right] .
\end{gathered}
$$

By ordering the elements of $\Omega$ lexicographically, we obtain the following transition probability matrix:

$$
P=\left(\begin{array}{cccccc}
A & B_{0} & \cdots & \cdots & \cdots & 0 \\
A & 0 & B_{1} & 0 & \cdots & 0 \\
A & 0 & 0 & B_{2} & \cdots & \vdots \\
\vdots & \vdots & \ddots & \ddots & \ddots & 0 \\
A & 0 & \cdots & \cdots & 0 & B_{m-1} \\
C & 0 & \cdots & \cdots & \cdots & 0
\end{array}\right),
$$

where the submatrices $A, B_{i}$, and $C$ are given by

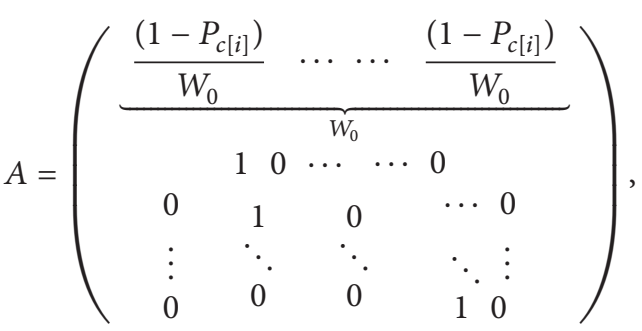

$$
\begin{aligned}
& B_{i}=\left(\begin{array}{cccc}
\frac{P_{c[i]}}{W_{i+1}} & \cdots & \cdots & \frac{P_{c[i]}}{W_{i+1}} \\
0 & \cdots & \cdots & 0 \\
\vdots & \cdots & \cdots & \vdots \\
0 & \cdots & \cdots & 0
\end{array}\right) \text {, }
\end{aligned}
$$

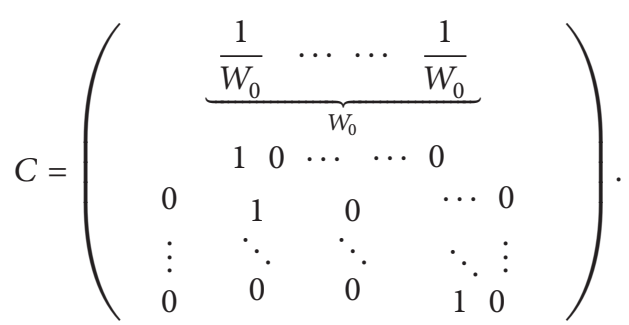

Note that the transition probability is a positive recurrent, and the system is stable. Therefore, there exist the 


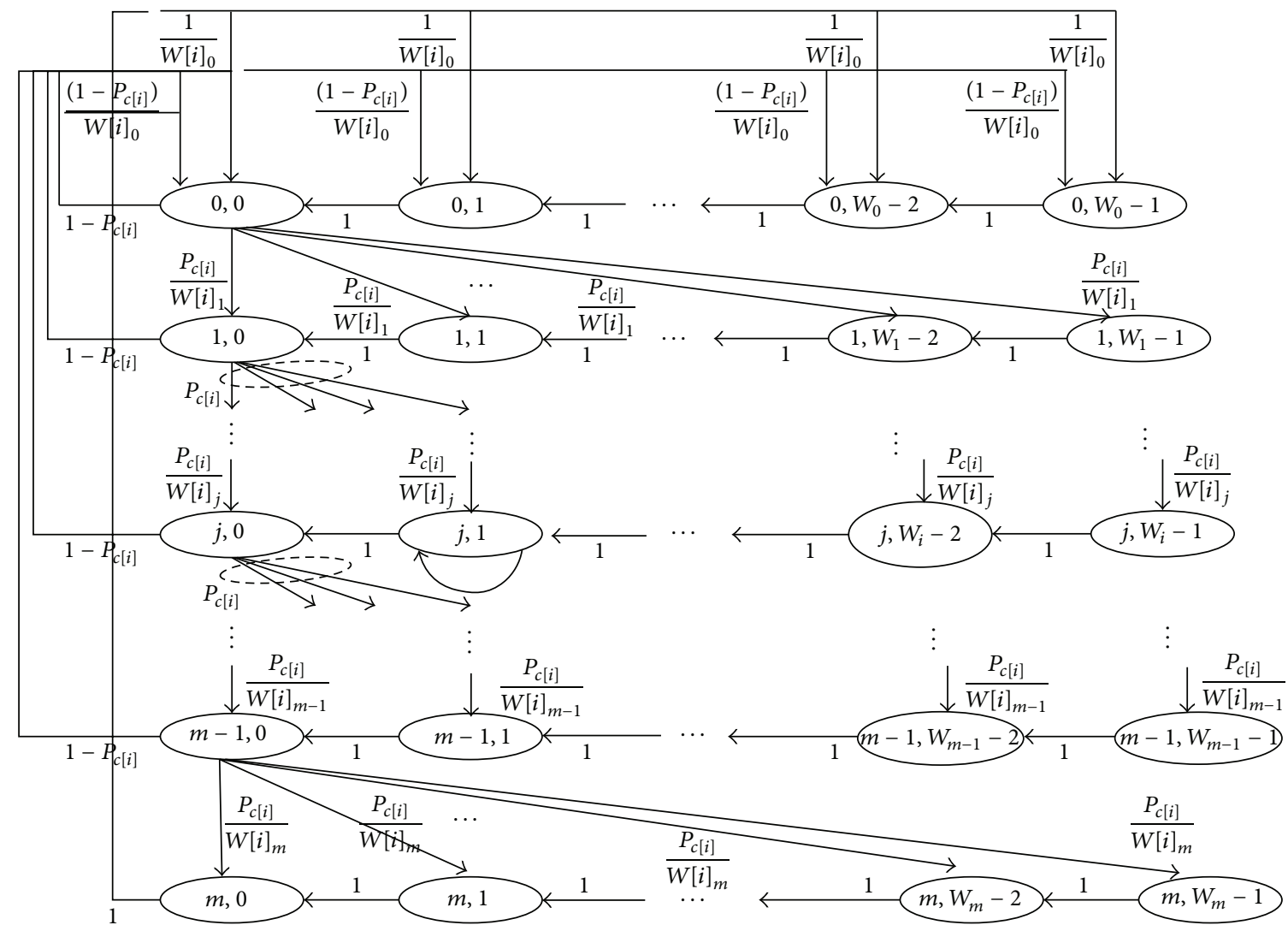

FIGURE 1: Markov chain model of EDCF in saturation regime.

stationary distributions $b_{j, k}$ of state $(j, k)$, which are given by $b_{j, k}=\lim _{t \rightarrow \infty} P\{s(t)=j, b(t)=k\}, j \in$ $[0, m], k \in\left[0, W[i]_{j}-1\right]$ satisfying $\mathbf{b} P=\mathbf{b}$, and $\mathbf{b e}=1$ exists, where $\mathbf{b}$ is the steady-state probability vector $\mathbf{b}=$ $\left(b_{0,0}, \ldots, b_{0, W_{0}-1}, \ldots, b_{m, 0}, \ldots, b_{m, W_{m}-1}\right)$, and $\mathbf{e}$ is the $1 \times(1+$ $\left.\sum_{i=0}^{m} W_{i}\right)$ column vector, for which the components consist of $1 \mathrm{~s}$.

By solving the balance equation $b_{j, k}=\sum_{\substack{l \neq, r \\ l \neq j, r \neq k}} p_{l, r} b_{l, r}$, we obtain the following equation:

$$
\begin{gathered}
b[i]_{j, k}=\frac{p_{c[i]}}{W_{j}} \cdot b[i]_{j-1,0}+b[i]_{j, k+1}, \\
j=1, \ldots, m, \quad k=0, \ldots, W_{j}-1 .
\end{gathered}
$$

Recursively solving (5), we obtain the following relations for the stationary probabilities:

$$
\begin{aligned}
b[i]_{j, 0} & =p_{c[i]}^{j} \cdot b[i]_{0,0}, \quad j=0, \ldots, m, \\
b[i]_{0, k} & =\frac{1-p_{c[i]}}{W_{0}} \sum_{j=0}^{m-1} b[i]_{j, 0}+\frac{1}{W_{0}} b[i]_{0,0} \\
& =\frac{1}{W_{0}} b[i]_{0,0}, \quad k=1, \ldots, W_{0}-1
\end{aligned}
$$

$$
\begin{array}{r}
b[i]_{j, k}=\frac{p_{c[i]}}{W_{j}}\left(W_{j}-k\right) b_{j-1,0}=\frac{W_{j}-k}{W_{j}} p_{c[i]}^{j} b[i]_{0,0}, \\
j=1, \ldots, m, \quad k=1, \ldots, W_{j}-1 .
\end{array}
$$

By using be $=1$ and $W_{j}=2^{j} W$ for $j=1, \ldots, m$, we calculate $b[i]_{0,0}$ as follows:

$$
\begin{aligned}
1= & \sum_{j=0}^{m} \sum_{k=0}^{W[i]_{j}-1} b[i]_{j, k} \\
= & \sum_{k=0}^{W[i]_{0}-1} b[i]_{0, k}+\sum_{k=0}^{W\left[i_{1}-1\right.} b[i]_{1, k}+\cdots+\sum_{k=0}^{W[i]_{m}-1} b[i]_{m, k} \\
= & b_{0,0}+\frac{p_{c[i]}}{W_{1}} b_{0,0} \sum_{k=0}^{W_{1}-1}\left(W_{1}-k\right)+\frac{p_{c[i]}^{2}}{W_{2}} b_{0,0} \\
& \times \sum_{k=0}^{W_{2}-1}\left(W_{2}-k\right)+\cdots+\frac{p_{c[i]}^{m}}{W_{m}} b_{0,0} \sum_{k=0}^{W_{m}-1}\left(W_{m}-k\right) \\
= & b_{0,0}\left\{1+\frac{1-p_{c[i]}^{m}}{2\left(1-p_{c[i]}\right)}+W_{0} p_{c[i]} \frac{1-\left(2 p_{c[i]}\right)^{m}}{1-\left(2 p_{c[i]}\right)}\right\} .
\end{aligned}
$$


Therefore, $b[i]_{0,0}$ is given by

$$
\begin{aligned}
b[i]_{0,0}=2( & \left.1-2 p_{c[i]}\right)\left(1-p_{c[i]}\right) \\
\times & \left(2\left(1-2 p_{c[i]}\right)\left(1-p_{c[i]}\right)\right. \\
& +\left(1-2 p_{c[i]}\right)\left(1-p_{c[i]}\right)^{m} \\
& \left.+W[i]_{0} p_{c[i]}\left(1-\left(2 p_{c[i]}\right)^{m}\right)\left(1-p_{c[i]}\right)\right)^{-1} .
\end{aligned}
$$

Substituting (10) into (6)-(8), we get the stationary probabilities, which are given by

$$
\begin{aligned}
& b[i]_{j, 0}=2\left(1-2 p_{c[i]}\right)\left(1-p_{c[i]}\right) p_{c[i]}^{j} \\
& \times\left(2\left(1-2 p_{c[i]}\right)\left(1-p_{c[i]}\right)\right. \\
& +\left(1-2 p_{c[i]}\right)\left(1-p_{c[i]}\right)^{m} \\
& \left.+W[i]_{0} p_{c[i]}\left(1-\left(2 p_{c[i]}\right)^{m}\right)\left(1-p_{c[i]}\right)\right)^{-1}, \\
& j=0, \ldots, m, \\
& b[i]_{0, k}=\frac{1}{W_{0}} \cdot 2\left(1-2 p_{c[i]}\right)\left(1-p_{c[i]}\right) \\
& \times\left(2\left(1-2 p_{c[i]}\right)\left(1-p_{c[i]}\right)\right. \\
& +\left(1-2 p_{c[i]}\right)\left(1-p_{c[i]}\right)^{m} \\
& \left.+W[i]_{0} p_{c[i]}\left(1-\left(2 p_{c[i]}\right)^{m}\right)\left(1-p_{c[i]}\right)\right)^{-1} \\
& k=1, \ldots, W_{0}-1 \text {, } \\
& b[i]_{j, k}=\frac{W_{j}-k}{W_{j}} \cdot 2\left(1-2 p_{c[i]}\right)\left(1-p_{c[i]}\right) p_{c[i]}^{j-1} \\
& \times\left(2\left(1-2 p_{c[i]}\right)\left(1-p_{c[i]}\right)\right. \\
& +\left(1-2 p_{c[i]}\right)\left(1-p_{c[i]}\right)^{m} \\
& \left.+W[i]_{0} p_{c[i]}\left(1-\left(2 p_{c[i]}\right)^{m}\right)\left(1-p_{c[i]}\right)\right)^{-1} \\
& j=1, \ldots, m, \quad k=1, \ldots, W_{j}-1 .
\end{aligned}
$$

We note that the transmission probability $\tau[i]$ and the collision probability of $p_{c[i]}$ are given by

$$
\begin{gathered}
\tau[i]=\sum_{j=0}^{m} b[i]_{j, 0}, \\
p_{c[i]}=1-(1-\tau[i])^{n[i]-1} \prod_{h=0, h \neq i}^{h=3}(1-\tau[h])^{n[h]},
\end{gathered}
$$

respectively, where $n[i]$ is the number of nodes that have packets to transmit in class $i$.

\section{Probability Distribution of MAC Layer Latency and Jitter}

Three basic processes occur when the MAC layer transmits a packet: decrement of the back-off counter that takes time period $\sigma$ (the time slot unit), successful packet transmission that takes time period $T[i]_{s}$, and packet collision that takes time period $T[i]_{c}$. We assume that $\sigma$ is a positive constant, and $T[i]_{s}$ and $T[i]_{c}$ are nonnegative random variables representing the period that the medium is sensed busy due to a successful transmission, and the period that the medium is sensed busy by each station due to a collision, respectively.

The MAC layer latency is the time interval from the time instant that a packet reaches the head of the queue and starts to contend for transmission to the time instant that either successful transmission of the packet is acknowledged, or the packet is dropped. On the other hand, the jitter is the interarrival time between successful packet transmissions of station and is obtained from the standard deviation of the latency.

These times are important for investigating the performance of higher protocol layers. Apparently, the MAC layer latency and jitter are discrete random variables, since the time unit used in transmission is a timeslot. Even though $T[i]_{s}$ and $T[i]_{c}$ depend on the transmission rate, the packet length, and the packet header overhead (a discrete unit, i.e. a bit), as well as the specific transmission scheme (basic access or RTS/CTS), we are assuming the variables take discrete values of the timeslot unit.

If the basic scheme is adopted, $T[i]_{c}$ is determined by the longest colliding packet. When the probability of three or more packets simultaneously colliding is neglected, its probability distribution can be approximated by the following equation:

$$
\begin{aligned}
\operatorname{Pr}\left\{T_{c}=i\right\}= & \operatorname{Pr}\left\{l_{1}=i, l_{2} \leq i\right\}+\operatorname{Pr}\left\{l_{2}=i, l_{1} \leq i\right\} \\
& -\operatorname{Pr}\left\{l_{1}=i, l_{2}=i\right\},
\end{aligned}
$$

where $l_{i}(i=1,2)$ is the packet length of the $i$ th colliding packet. In this mechanism, the time taken for a successful transmission is given by

$$
T[i]_{s}=\mathrm{DATA}+\mathrm{ACK}+2 \times \operatorname{SIFS}+\operatorname{AIFS}[i]
$$

while the time taken for a collision $T[i]_{c}$ is equal to

$$
T[i]_{c}=\mathrm{DATA}^{*}+\operatorname{AIFS}[i],
$$

where DATA* is the time taken for the longest frame involved in a collision. Thus we can infer that

$$
\begin{gathered}
G_{T[i]_{c}}(Z) \approx Z^{\mathrm{EIFS}} \frac{1}{\left(l_{\max }-l_{\min }+1\right)^{2}} \\
\times \sum_{k=l_{\min }}^{l_{\max }}\left(2 i-2 l_{\min }+1\right) Z^{k}, \\
G_{T[i]_{s}}(Z)=Z^{\mathrm{SIFS}+\mathrm{ACK}+\mathrm{AIFS}[i]} \frac{1}{l_{\max }-l_{\min }+1} \sum_{k=l_{\min }}^{l_{\max }} Z^{k} .
\end{gathered}
$$


For the RTS/CTS access mechanism, the period of a successful transmission $T[i]_{s}$ is equal to

$$
T[i]_{s}=\mathrm{RTS}+\mathrm{CTS}+\mathrm{DATA}+\mathrm{ACK}+3 \times \mathrm{SIFS}+\mathrm{AIFS}[i]
$$

while the period of a collision $T[i]_{c}$ is equal to

$$
T[i]_{\mathcal{C}}=\mathrm{RTS}+\mathrm{SIFS}+\mathrm{ACK}+\mathrm{AIFS}[i]=\mathrm{RTS}+\mathrm{EIFS} .
$$

Note that, except for the data component, all other factors on the right-hand side of (19) are constant, while all factors on the right-hand side of (20) are constant. Therefore, when the DATA length has a uniform distribution taking integers in the range $\left[l_{\max }, l_{\min }\right]$, the probability generation functions (PGF) of $T[i]_{s}$ and $T[i]_{c}$, which are denoted by $G_{T[i]_{s}}(Z)$ and $G_{T[i]_{c}}(Z)$, respectively, are given by

$$
\begin{aligned}
G_{T[i]_{s}}(Z)=E\left(Z^{T[i]_{s}}\right)= & Z^{\mathrm{RTS}+\mathrm{CTS}+\mathrm{ACK}+3 \mathrm{SIFS}+\mathrm{AIFS}[i]} \\
& \times \frac{1}{l_{\max }-l_{\min }+1} \sum_{i=l_{\min }}^{l_{\max }} Z^{i}, \\
G_{T[i]_{c}}(Z)= & E\left(Z^{T[i]_{c}}\right)=Z^{T\left[i_{c}\right.} .
\end{aligned}
$$

In the case that the DATA length is a fixed value $l_{D}$, its PGF $G_{T[i]_{s}}(Z)$ equals

$$
G_{T[i]_{s}}(Z)=Z^{\text {RTS+CTS }+ \text { ACK }+3 \operatorname{SIFS}+\mathrm{AIFS}[i]+l_{D}} .
$$

In the back-off process, if the medium is idle, the backoff counter is decreased by one, for every idle slot detected. During detection of an ongoing successful transmission, the back-off timer will be suspended and deferred for a time period $T[i]_{s}$, while if there are collisions between stations, the deferring time will be $T[i]_{c}$. Therefore, the latency $w[i]_{s}$, expressed as the time taken by the data link layer to successfully deliver a class $i$ packet over the radio channel, can be written as

$$
w[i]_{s}=T[i]_{s}+\sum_{j=1}^{n[i]_{c}} T[i]_{c, j}+\mathbb{T}[i]_{B}
$$

where $n[i]_{c}$ is the number of collisions the packet undergoes before success, taking time $T[i]_{c, j}$ at each collision, and $\mathbb{T}[i]_{B}$ is the contribution to the delay due to the backoff procedure. We assume that the transmission times are independent identically distributed (i.i.d.) random variables, with probability mass function $p_{T[i]_{s}}$. Note that the number of collisions $n[i]_{c}$ a packet undergoes before success will be a geometric random variable with probability mass function (PMF) $\operatorname{Pr}\left(n[i]_{c}=r\right)=p_{n\left[i_{c}\right.}(r)=\left(1-p_{c[i]}\right) p_{c[i]}^{r}$, where $p_{c[i]}$ is given in Section 2. Then the PGF for the latency $w[i]_{s}$ is given by

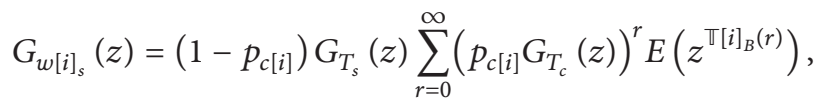

TABLE 1: DSSS system parameters and access category parameters used in simulation.

\begin{tabular}{lccc}
\hline Parameter & Value & Parameter & Value \\
\hline Channel bit rate & $1 \mathrm{Mbps}$ & AIFS [3] & 5 \\
Average frame size & $600 \mathrm{bits}$ & AIFS [2] & 3 \\
MAC header & 224 & AIFS [1] & 7 \\
PHY header & 192 & CWmin [4] & 7 \\
ACK & $112+$ PHY header & CWmin [3] & 15 \\
RTS & $160+$ PHY header & CWmin [2] & 31 \\
CTS & $112+$ PHY header & CWmin [1] & 31 \\
Slot time & $20 \mu \mathrm{sec}$ & CWmax [4] & 15 \\
SIFS & $10 \mu \mathrm{sec}$ & CWmax [3] & 15 \\
DIFS & $34 \mu \mathrm{sec}$ & CWmax [2] & 1023 \\
AIFS [4] & 2 & CWmax [1] & 1023 \\
\hline
\end{tabular}

TABLE 2: Comparisons between numerical and simulation results on latency (unit: sec).

\begin{tabular}{lccc}
\hline $\begin{array}{l}\text { The number of nodes } \\
\text { for each class }\end{array}$ & Class index & $\begin{array}{c}\text { Numerical } \\
\text { results }\end{array}$ & Simulation \\
\hline \multirow{4}{*}{3} & Class 4 & 1.2643 & 1.2643 \\
& Class 3 & 1.2005 & 1.2023 \\
& Class 2 & 1.0746 & 1.0701 \\
& Class 1 & 0.7321 & 0.7279 \\
\hline \multirow{3}{*}{4} & Class 4 & 1.338 & 1.3417 \\
& Class 3 & 1.2986 & 1.2944 \\
& Class 2 & 1.2198 & 1.2174 \\
& Class 1 & 1.0006 & 1.0032 \\
\hline \multirow{3}{*}{5} & Class 4 & 1.3983 & 1.4027 \\
& Class 3 & 1.3798 & 1.383 \\
& Class 2 & 1.3423 & 1.3383 \\
& Class 1 & 1.2361 & 1.2408 \\
\hline
\end{tabular}

where $\mathbb{T}[i]_{B}(r)$ is the overall back-off time, given that the packets experience $r$ collisions before success. It is worth stressing that, in the case of the basic access mode, $T[i]_{c}$ is statistically dependent on $T[i]_{s}$. However, in (25) we neglect this dependency and assume that $T[i]_{c, j}$ and $T[i]_{c}$ are independent. Such an assumption is clearly an approximation; however, it does not compromise the fidelity of the results, as proved by the comparison with the simulation results.

Let $\xi$ be the tick period, which is the time interval for one of the following three events; channel is idle, transmission success, and collision. Then it can be expressed as

$$
\xi=\sigma x_{I}+T[i]_{S} x_{S}+T[i]_{C} x_{C}
$$

where $x_{A}$ is an indicator function that takes the value 1 if event $A$ occurs, and 0 otherwise. Note that the back-off counter is decremented by 1 per time slot $\sigma$, and when any node undergoes transmission success or a collision, the back-off counter remains unchanged. Furthermore, in any tick period, the three events are pairwise disjoint. Let $\mathbb{\square}[i]_{B}(r)$ be the 
TABLE 3: Comparisons between numerical and simulation results on jitter (unit: sec).

\begin{tabular}{lccc}
\hline $\begin{array}{l}\text { The number of nodes } \\
\text { for each class }\end{array}$ & Class index & $\begin{array}{c}\text { Numerical } \\
\text { results }\end{array}$ & Simulation \\
\hline \multirow{4}{*}{3} & Class 4 & 0.6937 & 0.6937 \\
& Class 3 & 0.6801 & 0.6819 \\
& Class 2 & 0.6509 & 0.6463 \\
& Class 1 & 0.5457 & 0.5416 \\
\hline \multirow{4}{*}{4} & Class 4 & 0.709 & 0.7127 \\
& Class 3 & 0.7009 & 0.6967 \\
& Class 2 & 0.6834 & 0.6811 \\
& Class 1 & 0.6195 & 0.6227 \\
\hline \multirow{5}{*}{5} & Class 4 & 0.721 & 0.7255 \\
& Class 3 & 0.7174 & 0.7206 \\
& Class 2 & 0.7094 & 0.7053 \\
& Class 1 & 0.6797 & 0.6844 \\
\hline
\end{tabular}

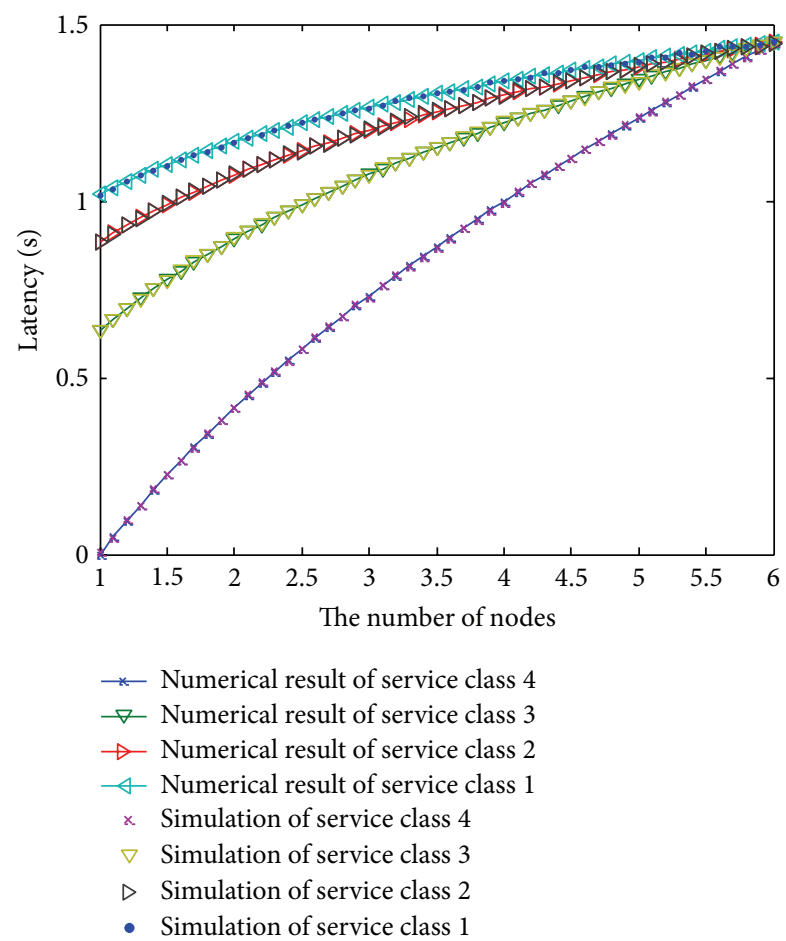

FIGURE 2: Latency result according to service classes.

overall back-off time, given that the packets experience $r$ collisions before success. Then it can be written as

$$
\mathbb{T}[i]_{B}(r)=\left\{\begin{array}{ll}
0 & \text { if } W[i]_{r}=0 ; \\
\sum_{j=1}^{W[i]_{r}} \xi[i]_{j} & \text { if } W[i]_{r}>0,
\end{array} \quad r=0,1, \ldots, m-1,\right.
$$

where $W[i]_{r}$ is the number of tick periods that a node waits before attempting the $r+1$ packet transmission, given that the first $r$ attempts resulted in collisions. Note that $\xi[i]_{j}$,

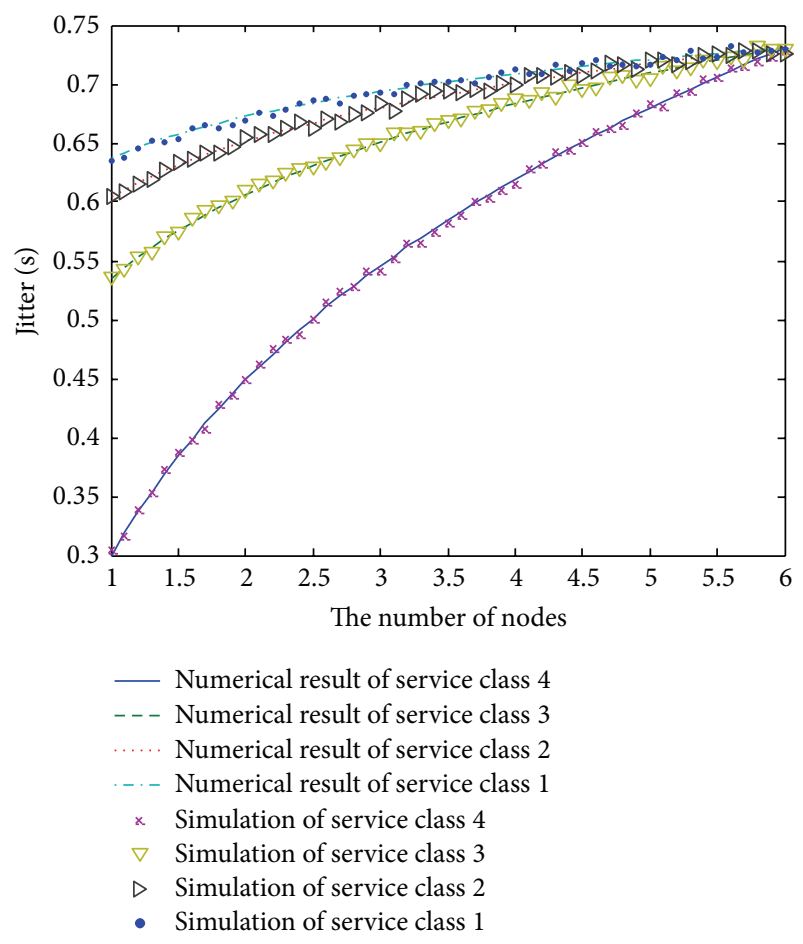

FIGURE 3: Jitter performance according to service classes.

$j=1, \ldots, W[i]_{m}$, is an i.i.d random variable, and its PGF, $G_{\xi}(z)$, is given by

$$
G_{\xi[i]}(z)=p_{S[i]} G_{T[i]_{s}}(z) \cdot p_{C[i]} G_{T[i]_{c}}(z) \cdot p_{I} z^{\sigma},
$$

where $p_{S[i]}$, $p_{I}$, and $p_{C[i]}$ are given as follows:

$$
\begin{gathered}
p_{S[i]}=(n[i]-1) \tau[i](n[i]-1)^{n[i]-1} \prod_{h=0, h \neq i}^{h=3}(1-\tau[h])^{n[h]}, \\
p_{I}=\prod_{h=0}^{h=3}(1-\tau[h])^{n[h]}, \\
p_{C[i]}=1-p_{I}-p_{S[i]} .
\end{gathered}
$$

To determine the statistic of the number $W[i]_{r}$ of tick periods a node spends in back-off, we introduce some further notations. Let $x_{i}$ be the number of tick periods a node spends in the back-off stage $j$. Then let $\left\{x_{j}\right\}_{j=1}^{m}$ be the set of i.i.d, random variables. Since the back-off counter can take any of the values in the back-off window $\left[0, \ldots, W[i]_{j}-1\right]$ with probability $1 / W_{i}$, the PGF of $x_{i}$ is given by

$$
G_{x_{i}}(z)=\sum_{k=0}^{W[i]_{j}-1} \frac{z^{k}}{W[i]_{j}}=\frac{1-z^{W[i]_{0} 2^{j}}}{(1-z) W[i]_{0} 2^{j}} .
$$

Since the random variable $W[i]_{r}$ can be expressed as

$$
W[i]_{r}=\sum_{j=0}^{m} x[i]_{j} .
$$


The PGF of $W[i]_{r}$ is calculated as

$$
G_{W[i]_{r}}(z)=\frac{\prod_{j=0}^{r}\left(1-z^{W\left[i j_{2} j^{j}\right.}\right)}{\left[W[i]_{0}(1-z) 2^{r / 2}\right]^{r+1}}, \quad r=0, \ldots, m .
$$

Then the PGF for the latency $w[i]_{s}$ is calculated as follows:

$$
\begin{aligned}
G_{w[i]_{s}}(z)= & \left(1-p_{c[i]}\right) G_{T[i]_{s}}(z) \\
& \times \sum_{r=0}^{m}\left(p_{c[i]} G_{T[i]_{c}}(z)\right)^{r} E\left(z^{\sum_{j=1}^{W[i]]_{r}} \xi_{j}}\right) \\
= & \left(1-p_{c[i]}\right) G_{T[i]_{s}}(z) \\
& \times \sum_{r=0}^{m}\left(p_{c[i]} G_{T[i]_{c}}(z)\right)^{r} G_{W[i]_{r}}\left(G_{\xi}(z)\right) .
\end{aligned}
$$

Taking the inverse discrete fourier transform (IDFT) of $G_{W[i]_{s}}(z)$, we can numerically obtain the PMF of the latency:

$$
p_{w_{s}}\left(T_{b}\right)=\frac{1}{\sqrt{N}} \sum_{z=0}^{N-1} G_{W[i]_{s}}(z) e^{+j 2 \pi n z / N},
$$

and thereafter, the cumulative distribution function (CDF), $F_{w_{s}}(t)=P\left[w_{s}>t\right]$.

Taking the standard derivation of $T_{b}$, we can obtain the jitter as $\sqrt{\operatorname{Var}\left(T_{b}\right)}$; if the contention window size is constant at every stage $r$, the variance of the latency is given by

$$
\begin{aligned}
\operatorname{Var}\left\{\bar{T}_{B}^{i}(r)\right\} & =\operatorname{Var}\left\{\sum_{j=1}^{r} \bar{T}_{B}^{j}+(r-1) T[i]_{c}\right\} \\
& =\operatorname{Var}\left\{r T^{* i}+(r-1) T[i]_{c}\right\} \\
& =\left(T^{* i}+T[i]_{c}\right)^{2} \frac{\left(1-p_{s[i]}\right)}{p_{s[i]}^{2}} \\
& =\left[\frac{\xi\left(W^{*}-1\right)}{2}+T[i]_{c}\right] \frac{\left(1-p_{s[i]}\right)}{p_{s[i]}^{2}} .
\end{aligned}
$$

In the case of the binary exponential back-off algorithm for each class $i$, we apply squared and cross-product terms. For conciseness, we omit here the intermediate steps and give the final expression of the jitter obtained after some algebra, because $T[i]_{c}$ is considered as constant:

$$
\begin{aligned}
\operatorname{Var}\left\{T_{B}^{i}(r)\right\} & \cong \operatorname{Var}\left\{\bar{T}_{B}^{i}(r)\right\} \\
& =\left[\frac{\xi\left(W[i]_{r}-1\right)}{2}+T[i]_{c}\right]^{2} \frac{\left(1-p_{s[i]}\right)}{p_{s[i]}^{2}} .
\end{aligned}
$$

\section{Numerical Analysis}

In this section, we compare the numerical and simulation results, in order to quantify the effect of the approximation introduced into the theoretical model. Our analytical approach is inspired by work on the analysis of the EDCF [11] but has some distinguishing features, for example, the latency and jitter characteristics of the AC in the EDCF are evaluated. The default values selected in the standard for EDCF parameters are summarized in Table 1 . In order to verify the accuracy of the analytical model, the comparisons of the average latency and jitter for varying numbers of nodes within each class are presented in Tables 2 and 3, respectively. As shown in the tables, the results of simulation are almost the same as those of numerical analysis. All simulation results in the tables are obtained with a $95.24 \%$ confidence rate (CR), which is calculated by the following:

$$
\mathrm{CR}_{T}=\left(1-\left|\frac{E\left[T_{\mathrm{anal}}-T_{\text {sim }}\right]}{E\left[T_{\text {anal }}\right]}\right|\right) \times 100 \%,
$$

where $T$ denotes the average latency or jitter.

Figure 2 shows the curves corresponding to the latency obtained with packets of constant length, and the number of the nodes in each class in the cluster. The figure clearly shows the service differentiation, for instance, the latency of packets in the highest priority class is distributed at low values, while that of packets in the lowest priority class is distributed at high values. Figure 3 shows the jitter obtained from the standard deviation of latency. The jitters are produced at less than the latency. The jitter of higher priority is less than that of lower priority. Both Tables 2 and 3 show that the numerical and simulation results are in agreement.

Figure 4 shows the probability mass functions of the latency for 4 categories of services. The figure shows that the latency PMF values are spread from 0 to 0.9 , according to priority; in other words, the values for the highest priority service are concentrated on the latency between 0.005 and 0.017 (Figure $4(a)$ ), those for the middle priority service are spread from 0.05 to 0.14 (Figures 4(b) and 4(c)), and those for the lowest priority service are widely spread from 0 to 0.9 (Figure $4(\mathrm{~d})$ ). This implies that the services are appropriately differentiated, according to priority. In addition, the values are discrete, which is because the times for successful transmission and collision are constant. When the number of nodes in each class is 4 , the mean latency is 1.173 seconds in Table 2. The IEEE specification recommended that the voice service delay should be no more than 0.2 seconds; and therefore, the delay in such an environment is higher than the recommended value. This implies that the access point has to restrict the number of nodes.

\section{Conclusions}

This paper presented a numerical model for the latency and jitter of 802.11e EDCF in saturation mode. The model accounts for both arbitrary interframe spaces and contention window differentiation mechanisms and considers both basic and RTS/CTS access mechanisms. Most importantly, it contains a probability distribution of the MAC packet latency, which is needed for $\mathrm{M} / \mathrm{M} / 1$ systems with small contention windows or very high operating loads and describes the standard deviation of the MAC packet latency. From this model, we can choose the available number of nodes that 


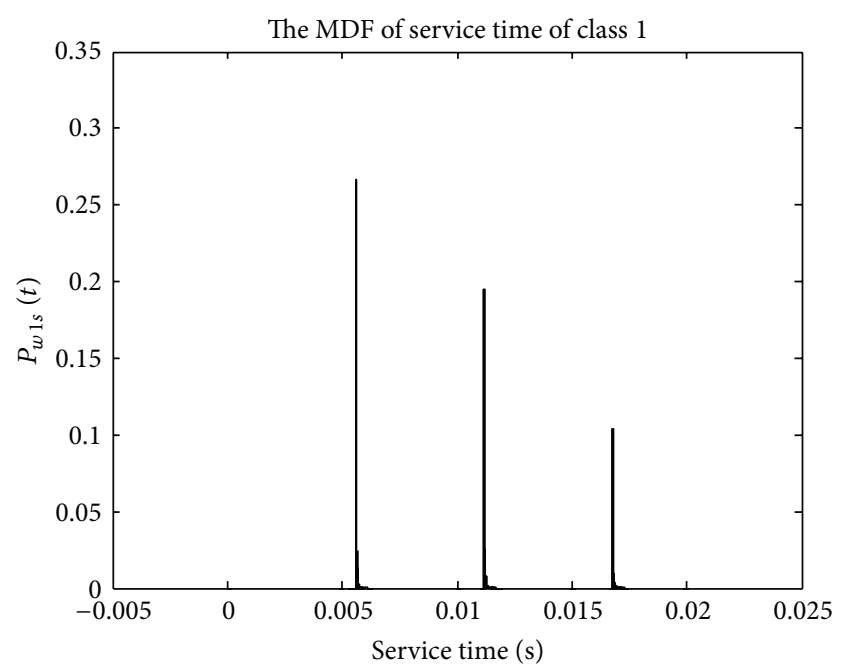

(a) Access category 4

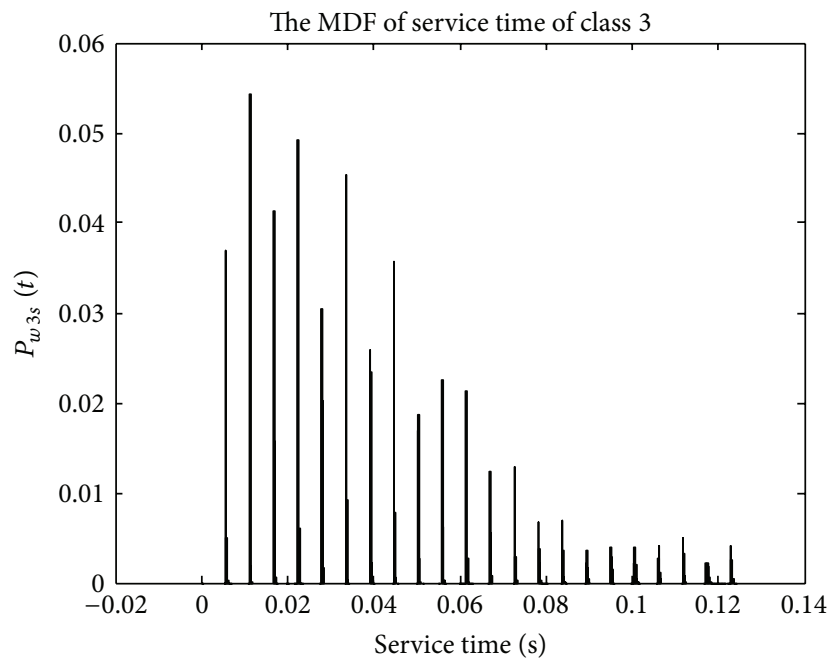

(c) Access category 2

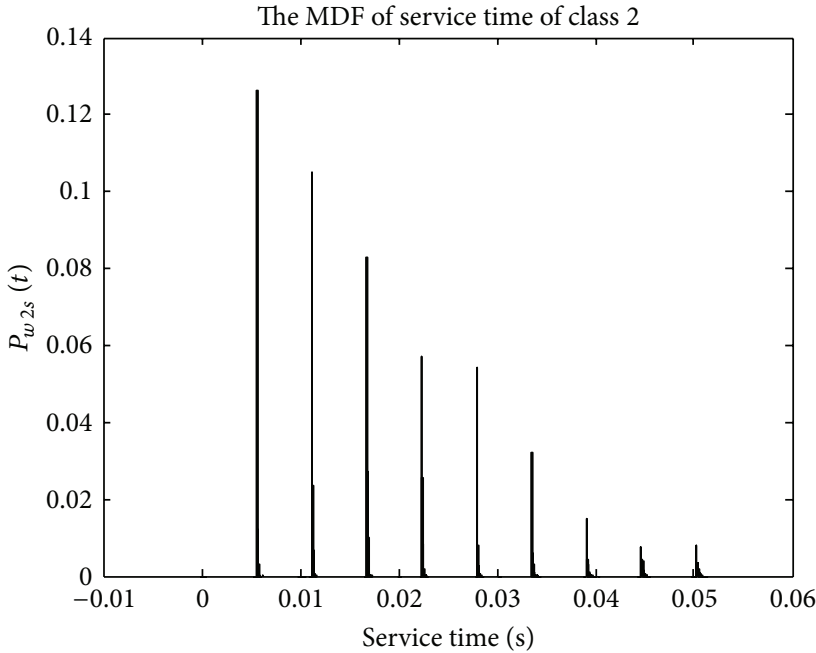

(b) Access category 3

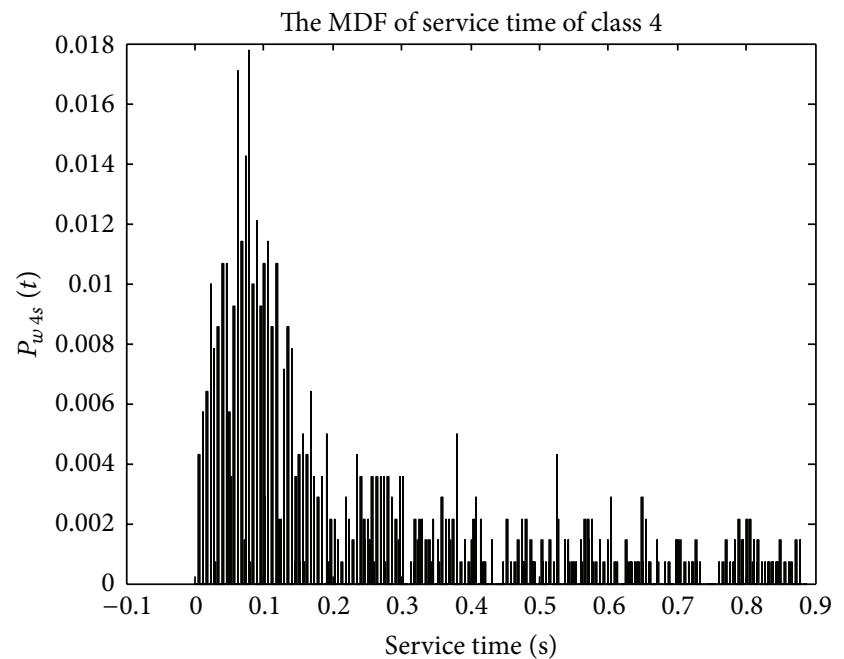

(d) Access category 1

Figure 4: Probability mass function.

determine the system performance, in order to satisfy user demand and handle the delay.

\section{Acknowledgment}

This research was supported by Hallym University Research Fund, 2013 (HRF-201309-002).

\section{References}

[1] Y. L. Hsu, Y. K. Huang, and A. C. Pang, "QoS enhancement for co-existence of IEEE 802.11e and legacy IEEE 802.11," in Proceedings of the IEEE Global Telecommunications Conference (GLOBECOM '08), pp. 1-5, New Orleans, La, USA, December 2008.

[2] S. Prahmkaew, "Performance evaluation of convergence ad hoc networks," Journal of Convergence, vol. 1, no. 1, pp. 101-106, 2011.

[3] K. Javed, U. Saleem, K. Hussain, and M. Sher, "An enhanced technique for vertical handover of multimedia traffic between
WLAN and EVDO," Journal of Convergence, vol. 1, no. 1, pp. 107112, 2011.

[4] N. Kryvinska, D. V. Thanh, and C. Strauss, "Integrated management platform for seamless services provisioning in converged network," International Journal of Information Technology, Communications and Convergence, vol. 1, no. 1, pp. 77-91, 2011.

[5] O. L. Sathappan, P. Chitra, P. Venkatesh, and M. Prabhu, "Modified genetic algorithm for multiobjective task scheduling on heterogeneous computing system," International Journal of Information Technology, Communications and Convergence, vol. 1, no. 2, pp. 146-158, 2011.

[6] H. Febiansyah and J. B. Kwon, "Generalized proxy-assisted periodic broadcasting (G-ProB) for heterogeneous clients in video-on-demand service," Journal of Information Processing Systems, vol. 6, no. 4, pp. 575-596, 2010.

[7] J. M. Oh and N. M. Moon, "A cultural dimensions model based on smart phone applications," Journal of Information Processing Systems, vol. 7, no. 1, pp. 209-220, 2011. 
[8] IEEE WG 802.11, Part 11: Wireless LAN Medium Access Control (MAC) and Physical layer (PHY) specifications, Amendment 8: Medium Access Control (MAC) Quality of Service Enhancements, IEEE Standards 802.11e, 2005.

[9] IEEE Std. 802.11-1999, Part 11: Wireless LAN Medium Access Control (MAC) and Physical Layer (PHY) specifications, Reference number ISO/IEC 8802-11:1999(E), IEEE Std. 802.11, 1999.

[10] G. Bianchi, "Performance analysis of the IEEE 802.11 distributed coordination function," IEEE Journal on Selected Areas in Communications, vol. 18, no. 3, pp. 535-547, 2000.

[11] J. W. Robinson and T. S. Randhawa, "Saturation throughput analysis of IEEE 802.11e enhanced distributed coordination function," IEEE Journal on Selected Areas in Communications, vol. 22, no. 5, pp. 917-928, 2004.

[12] J. Y. Lee and H. S. Lee, "A performance analysis model for IEEE 802.11e EDCA under saturation condition," IEEE Transactions on Communications, vol. 57, no. 1, pp. 56-63, 2009.

[13] H. Zhai, Y. Kwon, and Y. Fang, "Performance analysis of IEEE 802.11 MAC protocols in wireless LANs," Wireless Communications and Mobile Computing, vol. 4, no. 8, pp. 917-931, 2004.

[14] J. Mišić, S. Shafi, and V. B. Mišić, "The impact of MAC parameters on the performance of 802.15.4 PAN," Ad Hoc Networks, vol. 3, no. 5, pp. 509-528, 2005.

[15] E.-J. Kim, M. Kim, S.-K. Youm, S. Choi, and C.-H. Kang, "Priority-based service differentiation scheme for IEEE 802.15.4 sensor networks," AEU-International Journal of Electronics and Communications, vol. 61, no. 2, pp. 69-81, 2007.

[16] T.-Y. Kim, S. Youm, E.-J. Kim, and C.-H. Kang, "A service differentiation mechanism for improving the performance of IEEE 802.15.4 sensor networks," in Parallel and Distributed Processing and Applications, vol. 4330 of Lecture Notes in Computer Science, pp. 231-245, Springer, New York, NY, USA, 2006.

[17] N. C. Taher, Y. Ghamri-Doudane, B. E. Hassan, and N. Agoulmine, "An accurate analytical model for 802.11e EDCA under different traffic conditions with contention-free bursting," Journal of Computer Networks and Communications, vol. 2011, Article ID 136585, 24 pages, 2011. 


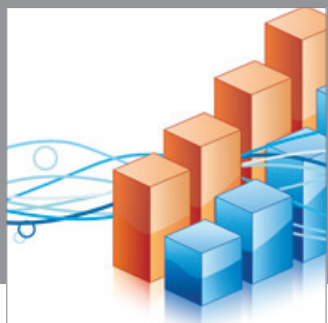

Advances in

Operations Research

mansans

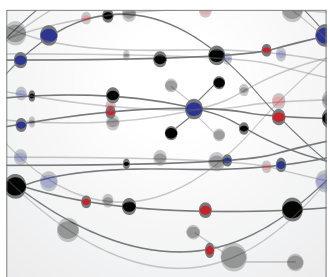

The Scientific World Journal
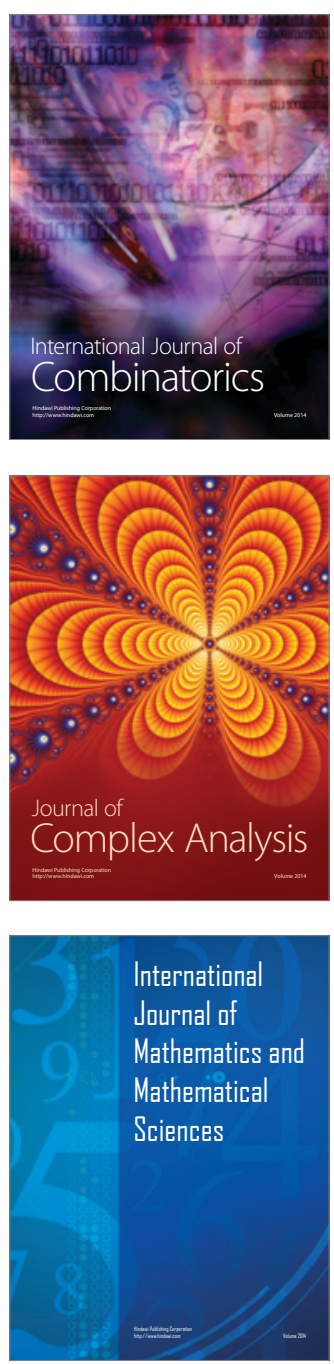
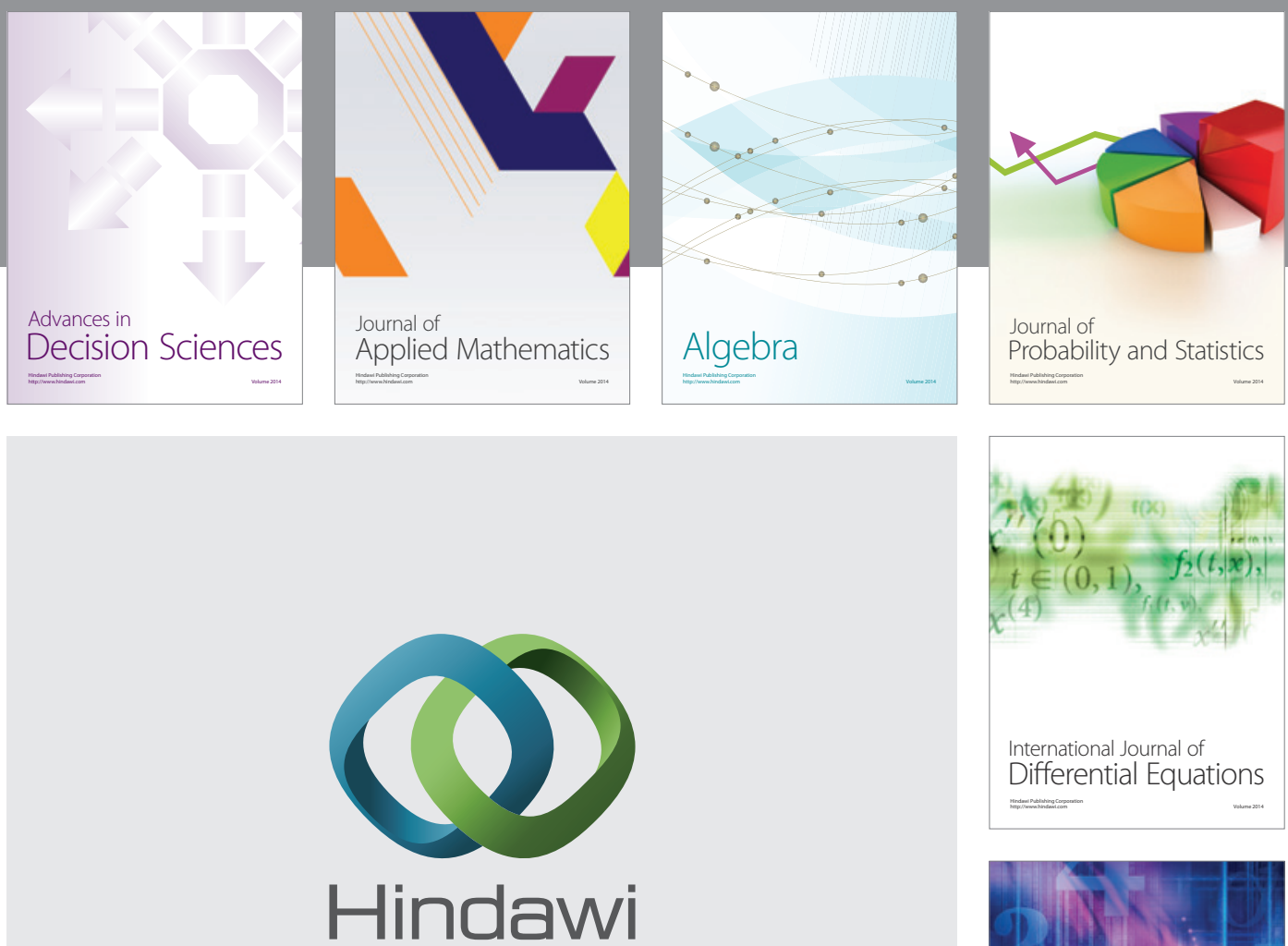

Submit your manuscripts at http://www.hindawi.com
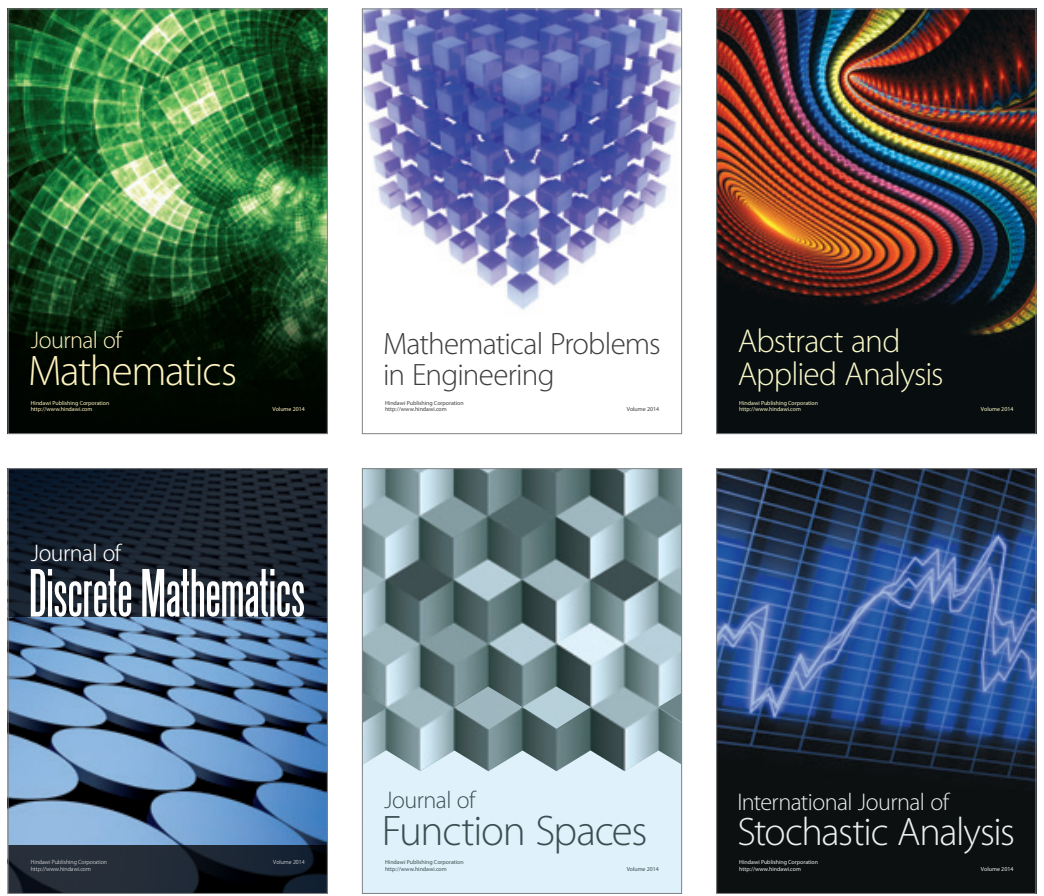

Journal of

Function Spaces

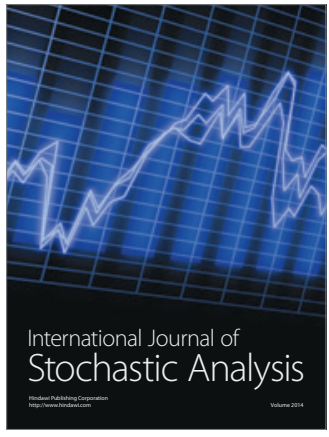

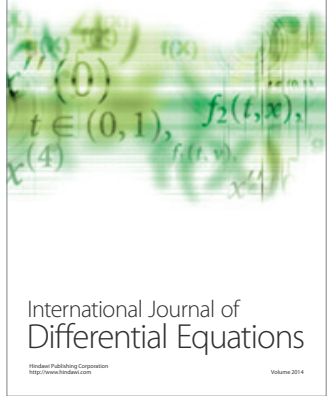
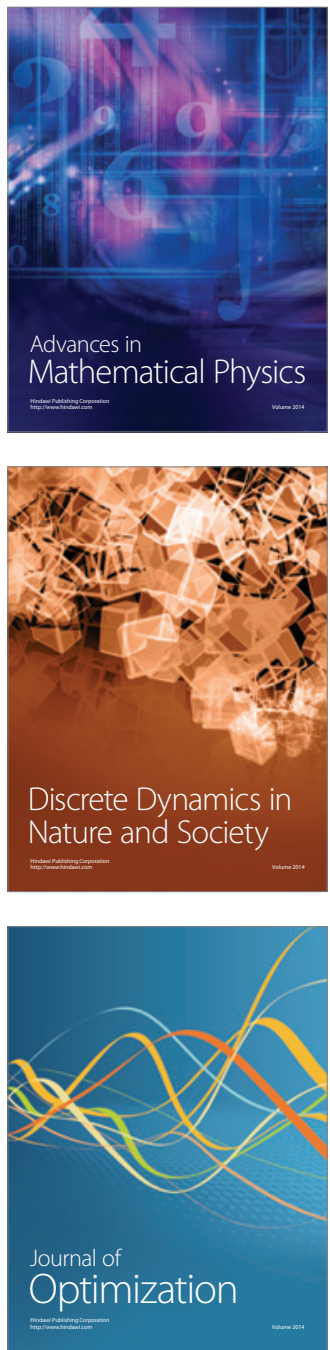